\title{
The development of AZD7624 for prevention of exacerbations in COPD: a randomized controlled trial
}

This article was published in the following Dove Press journal: International Journal of COPD

\author{
Naimish R Patel, ${ }^{1,2}$ Danen M \\ Cunoosamy,' Malin Fagerås,' \\ Ziad Taib,' Sara Asimus,' Tove \\ Hegelund-Myrbäck,' Sofia \\ Lundin,' Katerina Pardali,' \\ Nisha Kurian,' Eva Ersdal,' \\ Cecilia Kristensson,' Katarina \\ Korsback,' Robert Palmér,' \\ Mary N Brown, ${ }^{3}$ Steven \\ Greenaway, ${ }^{4}$ Leonard Siew, ${ }^{4}$ \\ Graham W Clarke, ${ }^{4,5}$ Stephen \\ I Rennard, ${ }^{6,7}$ Barry J Make, ${ }^{8}$ \\ Robert A Wise,' Paul Jansson' \\ 'Innovative Medicines and Early \\ Development, AstraZeneca, Gothenburg, \\ Sweden; ${ }^{2}$ Division of Pulmonary, \\ Critical Care, and Sleep Medicine, Beth \\ Israel Deaconess Hospital, Boston, \\ MA, ${ }^{3}$ Innovative Medicines and Early \\ Development, AstraZeneca, Boston, MA, \\ USA; ${ }^{4}$ Quintiles Drug Research Unit at \\ Guy's Hospital, London, ${ }^{5}$ Department \\ of Cardiothoracic Pharmacology, NHLI, \\ Imperial College London, London, UK; \\ ${ }^{6}$ Division of Pulmonary, Critical Care, \\ Sleep and Allergy, University of Nebraska, \\ Omaha, NE, USA; ${ }^{7}$ Clinical Discovery \\ Unit, Innovative Medicines and Early \\ Development, AstraZeneca, Cambridge, \\ UK; ${ }^{8}$ Division of Pulmonary Sciences and \\ Critical Care Medicine, National Jewish \\ Health, University of Colorado, Denver. \\ CO, ${ }^{9}$ Division of Pulmonary and Critical \\ Care, School of Medicine, Johns Hopkins \\ University, Baltimore, MD, USA
}

Correspondence: Paul Jansson AstraZeneca R\&D, Gothenburg,

SE-43I 83 Sweden

Tel +46708467353

Email paul.jansson@volvo.com
Background: p38 mitogen-activated protein kinase (MAPK) plays a central role in the regulation and activation of pro-inflammatory mediators. COPD patients have increased levels of activated p38 MAPK, which correlate with increased lung function impairment, alveolar wall inflammation, and COPD exacerbations.

Objectives: These studies aimed to assess the effect of p38 inhibition with AZD7624 in healthy volunteers and patients with COPD. The principal hypothesis was that decreasing lung inflammation via inhibition of $\mathrm{p} 38 \alpha$ would reduce exacerbations and improve quality of life for COPD patients at high risk for acute exacerbations.

Methods: The p38 isoform most relevant to lung inflammation was assessed using an in situ proximity ligation assay in severe COPD patients and donor controls. Volunteers aged 18-55 years were randomized into the lipopolysaccharide (LPS) challenge study, which investigated the effect of a single dose of AZD7624 vs placebo on inflammatory biomarkers. The Proof of Principle study randomized patients aged 40-85 years with a diagnosis of COPD for $>1$ year to AZD7624 or placebo to assess the effect of $\mathrm{p} 38$ inhibition in decreasing the rate of exacerbations.

Results: The p38 isoform most relevant to lung inflammation was p38 $\alpha$, and AZD7624 specifically inhibited $\mathrm{p} 38 \alpha$ and $\mathrm{p} 38 \beta$ isoforms in human alveolar macrophages. Thirty volunteers were randomized in the LPS challenge study. AZD7624 reduced the increase from baseline in sputum neutrophils and TNF- $\alpha$ by $56.6 \%$ and $85.4 \%$, respectively $(p<0.001)$. In the 213 patients randomized into the Proof of Principle study, there was no statistically significant difference between AZD7624 and placebo when comparing the number of days to the first moderate or severe exacerbation or early dropout.

Conclusion: Although p38 $\alpha$ is upregulated in the lungs of COPD patients, AZD7624, an isoform-specific inhaled p38 MAPK inhibitor, failed to show any benefit in patients with COPD.

Keywords: COPD, inflammation, p38 mitogen-activated protein kinase

\section{Plain language summary}

Despite optimal treatment, patients with COPD often experience exacerbations. Anti-inflammatory drugs such as inhaled corticosteroids can reduce the incidence of exacerbations. p38 mitogenactivated protein kinase (MAPK) plays a central role in the regulation and activation of key pro-inflammatory mediators, and COPD patients have increased levels of activated p38 MAPK in alveolar macrophages and other key inflammatory cells compared with cells from healthy smokers. A number of oral p38 inhibitors have been tested in COPD with a mix of background therapies. These studies have generally assessed lung function, not exacerbations, with no meaningful clinical improvement. In this set of studies, we identified the $\mathrm{p} 38 \alpha$ isoform as being 
activated in patients with severe COPD in both lung macrophages and epithelial cells. We also identified p38 $\alpha$ as the predominant isoform activated in the human alveolar macrophage response to LPS stimulation in vitro and AZD7624 as a specific inhibitor of $\mathrm{p} 38 \alpha$, with less activity against p38 $\beta$. AZD7624 decreased LPS-stimulated cytokine release in human alveolar macrophages in vitro, and decreased systemic and lung inflammatory markers after inhaled LPS challenge. However, AZD7624 failed to provide any benefit in a 3-month study of COPD exacerbations.

\section{Introduction}

Patients with COPD are susceptible to acute exacerbations at an increasing rate as the severity of COPD worsens, ${ }^{1}$ and frequent exacerbations are associated with an increased rate of lung function decline and death. ${ }^{2-4}$ Despite the current standard of care, a significant proportion of patients will continue to experience frequent exacerbations, ${ }^{5}$ and new drugs are needed to prevent exacerbations and reduce the associated morbidity in COPD. Exacerbations are associated with increased lung inflammation, in part because of increased bacterial or viral infections, or a combination of the two. ${ }^{6,7}$ The risk of exacerbations is correlated with markers of inflammation,,$^{8-10}$ and although the nature of the inflammatory response in COPD exacerbations is somewhat varied, it is usually associated with increased numbers of inflammatory cells and markers in the airways, as well as increased serum concentrations of fibrinogen and C-reactive protein (CRP). Anti-inflammatory drugs such as inhaled corticosteroids ${ }^{11}$ and roflumilast ${ }^{12}$ can decrease the incidence of exacerbations in COPD. Importantly, the addition of roflumilast to inhaled corticosteroids further reduces exacerbations, suggesting that anti-inflammatory agents work by complementary mechanisms and that additional benefit may result from novel anti-inflammatory approaches.

p38 mitogen-activated protein kinase (MAPK) plays a central role in the regulation and activation of key proinflammatory mediators. ${ }^{13,14}$ COPD patients have increased levels of activated p38 MAPK in alveolar macrophages and other key inflammatory cells compared with cells from healthy smokers. ${ }^{13}$ Furthermore, p38 MAPK activation correlates with the degree of lung function impairment and alveolar wall inflammation. ${ }^{15}$ Oral p38 inhibitors have been tested in COPD, but were assessed primarily for an effect on lung function rather than exacerbations. ${ }^{16,17}$ In addition, oral p38 inhibitors have been associated with systemic toxicities such as increased liver enzymes and rash. ${ }^{18}$ We used a step-wise approach of experiments to identify p $38 \alpha$ as the isoform most relevant to lung inflammation and developed an inhaled compound, AZD7624, to inhibit this isoform. The mechanism of p38 $\alpha$ inhibition with AZD7624 was then tested clinically in healthy volunteers via inhaled lipopolysaccharide (LPS) challenge. Finally, the principal hypothesis was evaluated: that decreasing lung inflammation via inhibition of $\mathrm{p} 38 \alpha$ would reduce exacerbations and improve quality of life for COPD patients at high risk for acute exacerbations.

\section{Methods}

\section{Study design and participants}

The in situ proximity ligation assay (isPLA) was used to delineate relevant p38 isoforms by measuring expression of $\mathrm{p} 38$ on lung tissue specimens obtained from nine COPD Global Initiative for Chronic Obstructive Lung Disease (GOLD) stage $\mathrm{IV}^{19}$ patients who underwent lung transplantation and four donor controls. p38 expression was measured in explanted lungs of patients with severe COPD to provide evidence that activated (phosphorylated) p38 is upregulated in patients with severe COPD. Post-surgical formalin-fixed paraffin-embedded (FFPE) lung tissue from the COPD patients and the four control donor lungs were sectioned at $2 \mu \mathrm{m}$, dried overnight at $37^{\circ} \mathrm{C}$, and rehydrated by incubating for $2 \times 5 \mathrm{~min}$ in xylene, $2 \times 1 \mathrm{~min}$ in $99 \%$ ethanol, $1 \mathrm{~min}$ in 95\% ethanol, $1 \mathrm{~min}$ in 70\% ethanol, $1 \mathrm{~min}$ in 50\% ethanol, and $5 \mathrm{~min}$ in double-distilled water. Antigen retrieval was performed by incubating the sections at $98^{\circ} \mathrm{C}$ for $47 \mathrm{~min}$ in a Tris- $\mathrm{HCl}$ buffer ( $\mathrm{pH}$ 7.8) containing 1 mM EDTA. All assessments were performed blind to the source of tissue and repeated on two separate occasions (further details in Supplementary material). Furthermore, alveolar macrophages, obtained from lung tissues of patients undergoing lung resection surgery by flushing with PBS, were incubated with $10 \mathrm{ng} / \mathrm{mL}$ LPS (\#L4516; Sigma Aldrich, St Louis, MO, USA) for $1 \mathrm{~h}$, pelleted, and fixed in formalin. The cell pellets were resuspended in Histogel ${ }^{\mathrm{TM}}$ (\#HG-4000-012; Thermo Fisher Scientific, Waltham, MA, USA), allowed to solidify, and processed for dehydration using standard methodology. The FFPE cell pellets were sectioned as above.

For the cytokine release assay, human alveolar macrophages were stimulated with LPS from Escherichia coli (Sigma) at a final concentration of $100 \mathrm{ng} / \mathrm{mL}$, and the cells were incubated for $18 \mathrm{~h}$ at $37^{\circ} \mathrm{C}$. Unstimulated cells were included in all assays as control. Following stimulation, the supernatants were collected, and tumor necrosis factor- $\alpha$ (TNF- $\alpha$ ) and IL-6 levels were determined by multiplex immunoassay from MSD ${ }^{\text {TM }}$ (Meso Scale Discovery, Rockville, MD, USA) (further details in Supplementary material).

Using a structure-based design, AZD7624, a potent and selective competitive inhibitor against $\mathrm{p} 38 \alpha / \beta$, was developed 
with predicted chemistry to support once-daily dosing and optimized for inhaled delivery.

A randomized controlled, double-blind, two-period, crossover LPS challenge study was undertaken in healthy volunteers as Proof of Mechanism to investigate the effect of AZD7624 vs placebo on inflammatory biomarkers in induced sputum and blood. Healthy male and female volunteers of non-childbearing potential aged 18-55 years were screened to ensure that adequate samples could be collected by sputum induction. ${ }^{20}$ In total, 30 volunteers were randomized to one of two treatment sequences (AZD7624 followed by placebo or vice versa) in a 1:1 ratio consisting of a single inhaled lung-deposited dose of AZD7624 $(1,200 \mu \mathrm{g})$ or placebo 30 min before LPS challenge administered via a SPIRA nebulizer (Spira Respiratory Care Center, Hameenlinna, Finland). For the LPS challenge, 45,000 endotoxin units of LPS was delivered by a breath-activated Mefar dosimeter. There was a minimum of a 28-day washout period between the treatment sequences. Sputum induction was performed $6 \mathrm{~h}$ post-challenge $(6.5 \mathrm{~h}$ post-dose $)$ for measurement of inflammatory biomarkers (Figure S1). Blood samples were collected $0.25,6.5,12$, and $24 \mathrm{~h}$ after AZD7624 or placebo dosing for the analysis of biomarkers. The first participant was enrolled on October 18, 2013, and the last participant's last visit was on April 29, 2014.

The COPD Proof of Principle study was a prospective, multicenter, randomized, double-blind, parallel-group, superiority trial vs placebo conducted at 39 centers in the USA, the Netherlands, South Africa, Argentina, Chile, and Peru. Between October 20, 2014, and December 11, 2015, participants between 40 and 85 years of age were recruited who had a diagnosis of COPD according to the GOLD 2014 guidelines for more than 1 year. Eligible participants had a post-bronchodilator ratio of $\mathrm{FEV}_{1}$ to $\mathrm{FVC}$ of $\leq 0.70$, an $\mathrm{FEV}_{1}$ after bronchodilators of $\leq 70 \%$ of predicted normal, ${ }^{21}$ a smoking history (current or former smoker) of at least 10 pack-years, and a history of two or more moderate or severe COPD exacerbations in the previous year (with at least one exacerbation on current maintenance therapy).

A seasonal recruitment strategy in the fall and winter months was used to recruit patients at a time when they were at highest risk of developing pulmonary exacerbations. ${ }^{22}$ Participants had to be stable on regular maintenance inhaler therapy, defined as at least an inhaled corticosteroid/longacting $\beta_{2}$-agonist combination in the previous 2 months and throughout the study. There was no restriction on additional COPD maintenance treatments such as long-acting muscarinic antagonists (LAMAs). Among the exclusion criteria were any exacerbation (defined as use of oral corticosteroids or antibiotics or hospital admission) related to COPD or respiratory infection within 6 weeks of randomization, and long-term use of oral steroids before and during treatment (apart from prescribed steroids at run-in and for treatment of an exacerbation). The Supplementary material lists all the exclusion criteria and drugs not allowed during the study. Participants meeting all eligibility criteria started a 2-week run-in period with oral steroids $(30 \mathrm{mg}$ /day oral prednisolone or equivalent) added to regular COPD maintenance therapy. The oral corticosteroid run-in was used to decrease intra- and inter-patient variability before randomization. ${ }^{23-25}$ After the run-in period, oral steroids were discontinued and the patients were randomly assigned to a 12-week treatment period in a 1:1 ratio to receive AZD7624 (1.0 mg delivered dose) or matching placebo administered once daily via two inhalations using a novel Aqueous Droplet Inhaler (Scandinavian Health AB, Nacka Strand, Sweden). Study schema are available in the Supplementary material (Figure S2).

The trials were approved by the ethics committee at each trial center (details provided in Supplementary material) and performed in accordance with the ethical principles that have their origin in the Declaration of Helsinki and that are consistent with the International Conference on Harmonization guidelines for Good Clinical Practice. All participants provided written informed consent.

\section{Outcomes}

The objectives of the Proof of Mechanism study in healthy volunteers were to investigate the effect of a single dose of inhaled AZD7624 on neutrophils (primary) and TNF- $\alpha$ concentration (secondary) in induced sputum after inhalation of LPS, compared with placebo (details in Supplementary material). The effect of inhaled AZD7624 on additional inflammatory biomarkers in induced sputum and blood after oral inhalation of LPS was evaluated as an exploratory objective. Safety was also evaluated.

The primary outcome of the Proof of Principle trial was time to first event of moderate or severe COPD exacerbations or early dropout related to worsening of COPD symptoms (composite endpoint referred to as "ExDo"). A moderate COPD exacerbation was defined as "worsening of COPD symptoms requiring treatment with systemic corticosteroid, antibiotics or both. ${ }^{26}$ A severe COPD exacerbation was defined as "worsening of COPD symptoms requiring hospital admission." Blinded adjudication of early dropout related to worsening of COPD symptoms was undertaken by the study team before database lock, 
and included discontinuations due to patient decision, worsening of COPD symptoms, lack of therapeutic effect, or patient lost to follow-up.

Secondary outcomes included moderate to severe exacerbations, lung function assessments $\left(\mathrm{FEV}_{1}, \mathrm{FVC}, \mathrm{FEV}_{1} / \mathrm{FVC}\right)$, and symptoms of COPD (St George's Respiratory Questionnaire for COPD Patients [SGRQ-C], Baseline Dyspnea Index/ Transition Dyspnea Index [BDI/TDI], and EXAcerbations of Chronic pulmonary disease Tool - Evaluating Respiratory Symptoms [EXACT E-RS ${ }^{\mathrm{TM}}$ ]). Blood biomarkers, of which high-sensitivity CRP, IL-6, and macrophage inflammatory protein-1 $\beta$ (MIP-1 $\beta$ ) are reported here, were also measured. Prespecified subgroup analyses included stratification of blood eosinophils into $\leq 2 \%$ or $>2 \%$ before oral corticosteroid run-in. Safety was also evaluated.

\section{Statistical analysis}

The human LPS challenge study was designed to randomize 26 volunteers to yield 20 volunteers with complete data, which corresponds to an $80 \%$ power to detect a $10.8 \%$ change from baseline in sputum neutrophils between AZD7624 and placebo at the $5 \%$ one-sided significance level. The expected within-volunteer SD was set to $12.5 \%$. It was assumed that about $25 \%$ of the randomized volunteers would have one period with no data owing to dropouts. Analyses of percentage change in neutrophils and TNF- $\alpha$ were performed using a covariance model with fixed effects for treatment and sequence, and neutrophil percentage differential (or TNF- $\alpha$ ) at baseline as a continuous covariate.

In the COPD Proof of Principle study, the randomization codes were computer generated by AstraZeneca R\&D using the validated AZ Global Randomization system (GRand) and loaded into an Interactive Voice Response System/ Interactive Web Response System (IVRS/IWRS) database. Randomization codes were generated in blocks to ensure approximate balance (1:1) between the two treatment arms. The randomization was stratified by country and use of LAMA maintenance treatment. The IVRS/IWRS allocated randomization codes sequentially within each center as patients became eligible for randomization. For each patient randomized, the IVRS/IWRS provided the investigator with a unique Kit ID number matching the treatment arm assigned to the patient.

The primary and safety analysis for the COPD Proof of Principle study was conducted with data from a modified intention-to-treat population, which included all participants who received at least one dose of study drug. It was assumed that $56 \%$ of participants in the control group would be exacerbation free during the study ( 3 months), and with
$80 \%$ power to detect a $45 \%$ reduction in hazard rate (time to first event of ExDo and time to first exacerbation) compared with the control group, this led to a sample size estimate of 106 patients per treatment arm with the addition of a conservative group sequential approach with one interim analysis. A reduction of $45 \%$ in hazard rate at 3 months is assumed to correspond to a $25 \%$ reduction in hazard rate at 12 months. ${ }^{27}$ For the primary analysis, a Cox proportional hazard model was fitted with treatment arm, country, LAMA maintenance treatment, age, and gender as covariates. Results are reported as the HR, 95\% CI, and $p$-value. Time to first ExDo event is presented as a Kaplan-Meier plot. The secondary endpoint of time to event of moderate or severe exacerbation was analyzed by a Cox proportional hazard model, and the rate of moderate and severe exacerbations was determined by the negative binomial method using the same covariates. Other secondary endpoints, including $\mathrm{FEV}_{1}$, FVC, FEV ${ }_{1}$ FVC, SGRQ-C, BDI/ TDI, EXACT E-RS, and night-time awakenings, were analyzed using a mixed-effects repeated measures model, with treatment, country, LAMA maintenance treatment, and visit as fixed effects; patient as a random effect; and baseline vales as a continuous covariate. Further details of the statistical analysis are available in the Supplementary material.

These trials are registered with Clinica Trials.gov (ClinicalTrials.gov identifier: NCT01937338 and NCT02238483).

\section{Role of the funding source}

The Proof of Mechanism trial in healthy volunteers was funded and designed by AstraZeneca and Quintiles. The COPD Proof of Principle trial was funded and designed by AstraZeneca and the academic partners. In the Proof of Principle study, AstraZeneca performed trial monitoring and reporting, provided oversight, and verified key results provided from the statistical analyses, which were performed by Quintiles on behalf of, and with oversight by, employees of AstraZeneca. A Data Monitoring Committee (for membership, see Supplementary material), independent of the study team, monitored safety throughout the study. The corresponding author had full access to all the data in the study and had final responsibility for the decision to submit for publication.

\section{Results}

Preclinical selection of $\mathrm{p} 38$ isoform target Using isPLA, we observed elevation of activated (phosphorylated) p38 $\alpha$ MAPK associated with alveolar macrophages (Figure 1A) and bronchial epithelium (Table S1) in lung tissue sections from COPD patients compared with samples from healthy controls. Following exposure of in vitro 


\section{A \\ A}

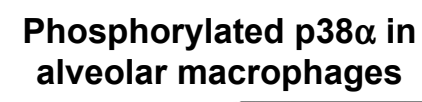

Phosphorylated $p 38 \alpha$ in alveolar macrophages

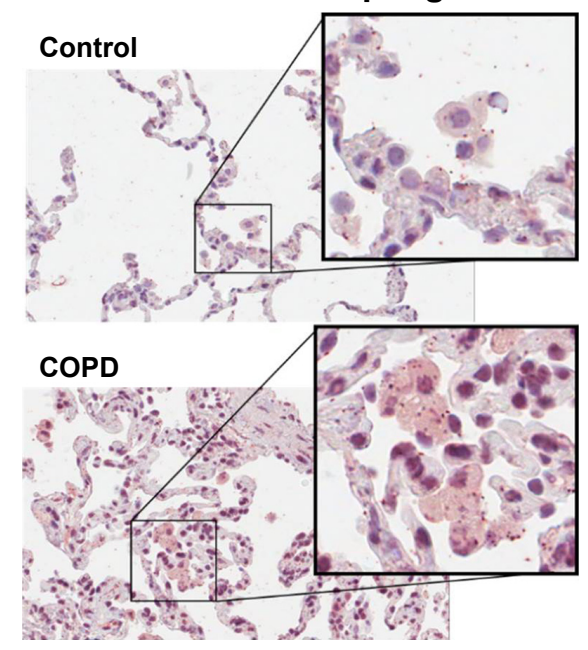

C

Attenuation of sputum neutrophil \% differential

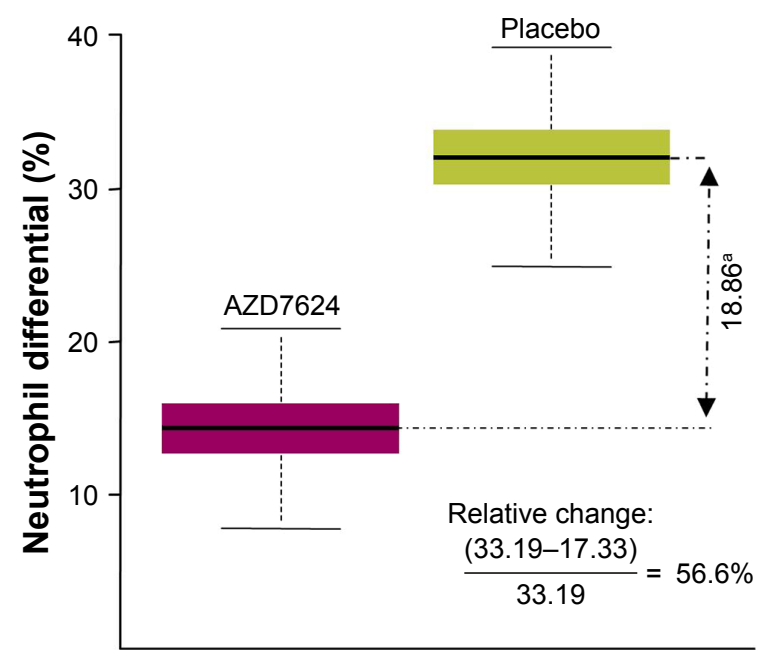

B Inhibition TNF- $\alpha$ in
alveolar macrophages

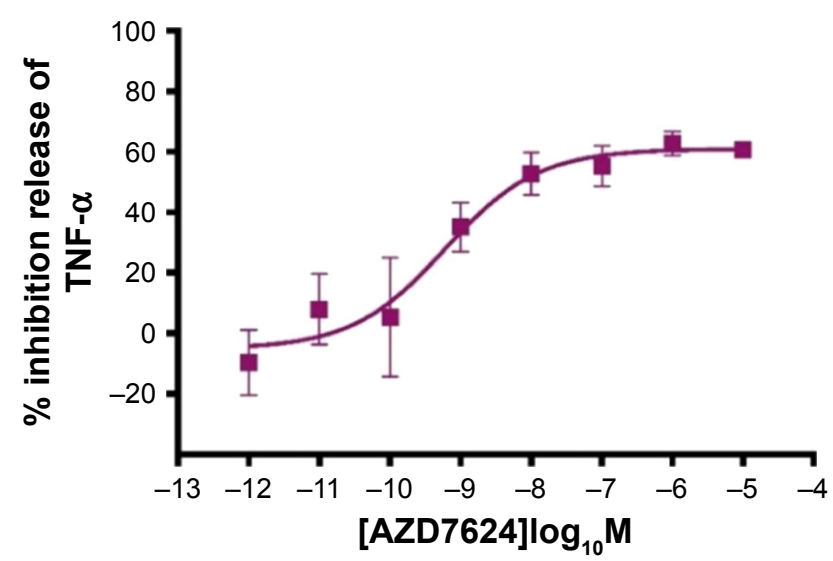

D Inhibition of TNF- $\alpha$ in sputum

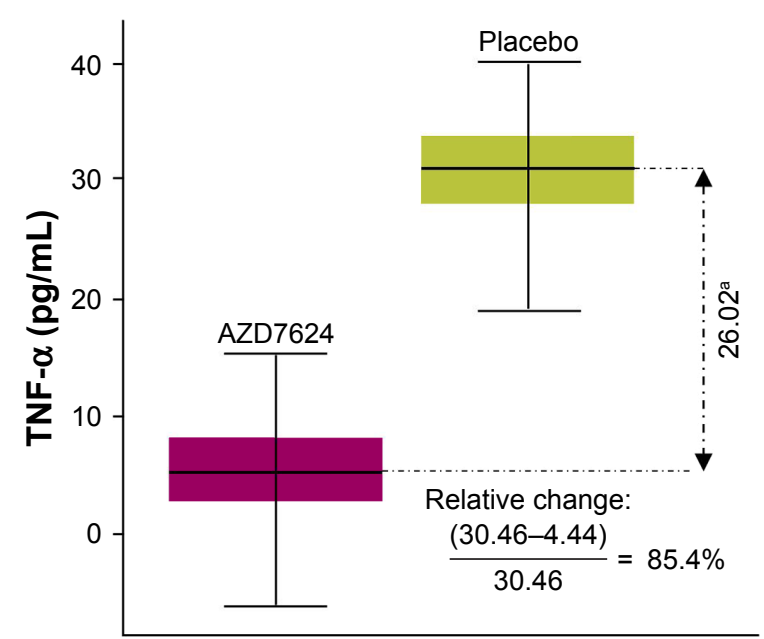

Figure I (A) isPLA of phosphorylated p38 $\alpha$ in alveolar macrophages of lung tissue from COPD patients and controls. Data are representative of two experiments. (B) AZD7624 inhibition of LPS-stimulated pro-inflammatory cytokine TNF- $\alpha$ in human alveolar macrophages. Data are presented as mean \pm SEM of $n=4$. AZD7624 attenuation of (C) sputum neutrophil \% differential and (D) TNF- $\alpha$ changes from baseline compared with placebo in the human LPS challenge study. Data are presented as mean \pm SEM of 24-27 subjects per treatment group. ${ }^{a} p<0.00$ I vs placebo.

Abbreviations: isPLA, in situ proximity ligation assay; LPS, lipopolysaccharide; SEM, standard error of the mean; TNF- $\alpha$, tumor necrosis factor- $\alpha$.

alveolar macrophages to LPS, there were marked increases in the total levels of $\mathrm{p} 38 \beta$ and p38 $\gamma$ MAPK isoforms, while p38 $\alpha$ MAPK was the only isoform that displayed increased phosphorylation (Figure S3). AZD7624, tested against $\alpha, \beta$, $\gamma$, and $\delta$ isoforms of human p38 MAPK, achieved a 15 -fold selectivity for $\mathrm{p} 38 \alpha$ vs $\mathrm{p} 38 \beta \mathrm{MAPK}$ and $>10,000$-fold selectivity vs p38 $\gamma$ and $\mathrm{p} 38 \delta$ MAPK (Table S2). In human alveolar macrophages, AZD7624 produced concentration-dependent inhibition of LPS-induced TNF- $\alpha$ (Figure 1B) and IL-6 (Figure S4) release, with negative log half-maximal inhibitory concentration $\left(\mathrm{pIC}_{50}\right)$ values of $-9.2 \pm 0.4$ and $-8.8 \pm 0.7$, respectively.

\section{Clinical Proof of Mechanism}

In the healthy volunteer LPS challenge study, 21 of 30 subjects randomized completed the study. Six discontinuations were due to insufficient sputum samples. The baseline demographic characteristics of the study population can be found in Table S3. AZD7624 reduced the increase from baseline in sputum neutrophil percentages and TNF- $\alpha$ following LPS challenge by $56.6 \%(p<0.001)$ (Figure 1C) and $85.4 \%(p<0.001)$ (Figure 1D), respectively. AZD7624 also significantly reduced additional inflammatory biomarkers from blood (neutrophil percentage, IL-6, MIP-1 $\beta$, and CRP) and sputum (IL-6, IL-8, and MIP-1 $\beta$ ) (Table S4). 
Table I Adverse events, Proof of Mechanism study ${ }^{\mathrm{a}}$

\begin{tabular}{|c|c|c|c|}
\hline Variable $^{b}$ & AZD7624 & Placebo & Total \\
\hline & $(n=27)$ & $(n=24)$ & $(\mathbf{N}=\mathbf{3 0})$ \\
\hline Participants with any $\mathrm{AE}$ & $6(22.2)$ & $9(37.5)$ & $13(43.3)$ \\
\hline Gastrointestinal disorders & I (3.7) & 0 & I (3.3) \\
\hline Dry mouth & I (3.7) & 0 & I (3.3) \\
\hline $\begin{array}{l}\text { General disorders and } \\
\text { administration site conditions }\end{array}$ & 0 & $3(12.5)$ & $3(10.0)$ \\
\hline Influenza-like illness & 0 & I (4.2) & I (3.3) \\
\hline Pyrexia & 0 & $2(8.3)$ & $2(6.7)$ \\
\hline Infections and infestations & $2(7.4)$ & $2(8.3)$ & $4(13.3)$ \\
\hline Folliculitis & 0 & I (4.2) & I (3.3) \\
\hline Upper respiratory tract infection & $2(7.4)$ & I (4.2) & $3(10.0)$ \\
\hline $\begin{array}{l}\text { Injury, poisoning, and procedural } \\
\text { complications }\end{array}$ & 0 & I (4.2) & I (3.3) \\
\hline Arthropod bite & 0 & $\mathrm{I}(4.2)$ & $\mathrm{I}(3.3)$ \\
\hline Nervous system disorders & I (3.7) & $2(8.3)$ & $3(10.0)$ \\
\hline Dizziness & 0 & I (4.2) & I (3.3) \\
\hline Headache & 0 & I (4.2) & I (3.3) \\
\hline Presyncope & I (3.7) & 0 & I (3.3) \\
\hline
\end{tabular}

Notes: Data are shown as number (\%) of participants. ${ }^{2}$ The safety analysis included patients who received a drug during the treatment period. ${ }^{b}$ Events were coded according to preferred terms in MedDRA, a standardized dictionary for clinical trials. ${ }^{38}$

Abbreviation: $\mathrm{AE}$, adverse event.
The incidence of adverse events (AEs) was similar across treatment groups, and the most commonly reported $\mathrm{AE}$ was upper respiratory tract infection (Table 1). Overall, 13 $(43.3 \%)$ of the 30 volunteers reported at least one AE. Six (22.2\%) of the 27 volunteers who received AZD7624 reported at least one $\mathrm{AE}$, while nine (37.5\%) of the 24 volunteers who received placebo reported at least one AE. One participant on placebo discontinued the study because of an $\mathrm{AE}$ of severe pyrexia in the first treatment sequence. No deaths or serious adverse events (SAEs) were reported in this study.

\section{Clinical Proof of Principle}

Of the total 327 participants screened for the study, 213 were randomized and 114 were not, mainly because of screening failure (Figure 2). Eighty-six participants came from the northern hemisphere (USA and the Netherlands), and 126 participants came from the southern hemisphere (Argentina, Peru, Chile, and South Africa). Baseline patient characteristics were similar between the two treatment arms (Table 2).

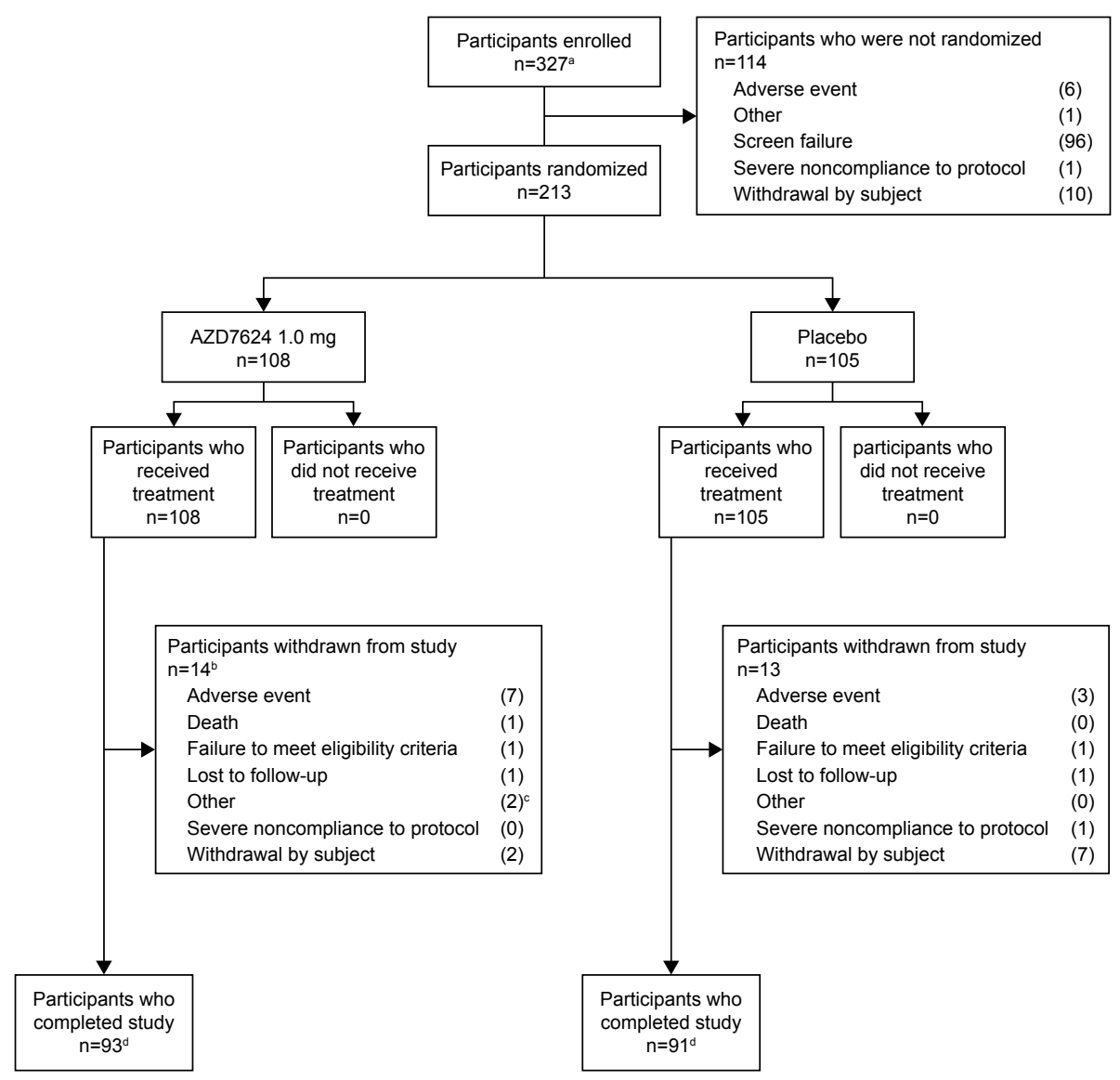

Figure 2 Patient disposition. anformed consent received. 'One patient was excluded from the analysis following review of protocol deviations owing to a lack of source data and Good Clinical Practice compliance. 'Patient withdrawal recorded as "other" by study site investigators; one patient was unable to complete the study visits owing to terminal illness of family member; another patient was withdrawn by the study site investigator after a reassessment of patient eligibility (on day 4 after randomization). ${ }^{\mathrm{d} C}$ Completion status of two patients was not recorded. 
Table 2 Baseline demographics, COPD Proof of Principle study

\begin{tabular}{|c|c|c|}
\hline Characteristic & $\begin{array}{l}\text { AZD7624 } \\
\frac{1.0 \mathrm{mg}}{(\mathrm{n}=107)^{\mathrm{d}}}\end{array}$ & Placebo \\
\hline Age (years) & $65.4 \pm 8.7$ & $64.2 \pm 8.7$ \\
\hline Female gender & $40(37.4)$ & $36(34.3)$ \\
\hline BMI $\left(\mathrm{kg} / \mathrm{m}^{2}\right)$ & $27.9 \pm 5.9$ & $27.5 \pm 5.4$ \\
\hline \multicolumn{3}{|l|}{ Ethnicity } \\
\hline White & $85(79.4)$ & $88(83.8)$ \\
\hline Black & $7(6.5)$ & $2(1.9)$ \\
\hline Native American & $3(2.8)$ & $4(3.8)$ \\
\hline Other & $12(1 \mathrm{I} .2)$ & II (I0.5) \\
\hline Peripheral oxygen saturation (\%) & $95.6 \pm 2.2$ & $95.9 \pm 2.0$ \\
\hline Former smoker & $66(61.7)$ & $73(69.5)$ \\
\hline Pre-bronchodilator FEV, (L) & $1.14 \pm 0.48$ & $1.15 \pm 0.46$ \\
\hline Post-bronchodilator FEV $(\mathrm{L})$ & $1.22 \pm 0.49$ & $1.29 \pm 0.50$ \\
\hline Predicted post-bronchodilator FEV & $44.5 \pm 15.6$ & $44.5 \pm 14.9$ \\
\hline \multicolumn{3}{|l|}{ COPD severity (GOLD) ${ }^{a}$} \\
\hline GOLD 2 & $22(20.6)$ & $20(19.0)$ \\
\hline GOLD 3 & $49(45.8)$ & $46(43.8)$ \\
\hline GOLD 4 & $35(32.7)$ & $39(37.1)$ \\
\hline \multicolumn{3}{|l|}{ Exacerbations in the past 12 months } \\
\hline 2 & $79(73.8)$ & $81(77.0)$ \\
\hline 3 & $21(19.6)$ & II (I0.5) \\
\hline$>3$ & $6(5.6)$ & II (I0.5) \\
\hline SGRQ-C ${ }^{b}$ & $56.5 \pm 0.1$ & $60.4 \pm 18.4$ \\
\hline $\mathrm{BDI}^{\mathrm{c}}$ & $5.3 \pm 2.2$ & $5.6 \pm 2.0$ \\
\hline Concomitant LAMA & $4 \mid(38.3)$ & $40(38.1)$ \\
\hline
\end{tabular}

Notes: Data are shown as mean \pm SD or $\mathrm{n}(\%)$. ${ }^{\text {TT The severity of COPD was }}$ determined on the basis of the 2015 GOLD grading of airflow limitation in patients with $\mathrm{FEV} / \mathrm{FVC}<0.70$ using post-bronchodilator spirometry, based on which GOLD 2: moderate with $50 \%<\mathrm{FEV}_{1}<80 \%$ of predicted, GOLD 3: severe with $\mathrm{FEV}_{1}<50 \%$ predicted, and GOLD 4: very severe with $\mathrm{FEV},<30 \%$ predicted. 'bScores on the SGRQ-C range from 0 to 100 , with higher scores indicating worse health status. ${ }^{\text {TT }}$ The $\mathrm{BDI}$ assesses breathlessness in patients with COPD. Scores range from 0 to 12. The lower the score, the worse the severity of dyspnea. ${ }^{d}$ One patient was excluded from the analysis following a review of protocol deviations owing to a lack of source data and Good Clinical Practice compliance.

Abbreviations: BDI, Baseline Dyspnea Index; BMI, body mass index; GOLD, Global Initiative for Chronic Obstructive Lung Disease; LAMA, long-acting muscarinic antagonist; SGRQ-C, St George's Respiratory Questionnaire for COPD Patients.

\section{Primary results}

No statistically significant difference between AZD7624 and placebo was observed when comparing the number of days to the first ExDo event (HR $=1.39,95 \%$ CI 0.81, 2.40) (Figure 3A). Similarly, no statistically significant difference was seen when analyzing the number of days to the first exacerbation (Figure 3B). Overall, there was a trend toward more exacerbations in the group treated with AZD7624. As detailed in Table 3, there was no statistically significant difference in change from baseline comparing the AZD7624 and placebo arms with respect to $\mathrm{FEV}_{1}, \mathrm{FVC}$, quality of life (SGRQ-C), dyspnea index (TDI), symptoms (as defined by EXACT E-RS), use of reliever medications, or night-time awakenings. In the predefined subgroups of baseline blood eosinophils, AZD7624 performed worse in participants with $>2 \%$ eosinophils (HR $=2.20,95 \%$ CI 0.95 , $5.09, p=0.07)$ than in patients with $\leq 2 \%(\mathrm{HR}=0.93,95 \% \mathrm{CI}$ $0.42,2.05 ; p=0.85$ ) (Figure 3C and D). The lack of treatment effect of AZD7624 on exacerbations was also observed in subgroups stratified by country, baseline $\mathrm{FEV}_{1}$, eosinophils, CRP, IL-6, fibrinogen, smoking status, bronchodilator response, or presence of chronic bronchitis.

\section{Secondary results}

No statistically significant difference was observed in any of the secondary efficacy endpoints between AZD7624 and placebo (Table 3). At study end, there was no statistically significant difference between AZD7624 and placebo in CRP, IL-6, and MIP-1 $\beta$ (Table S5). In the placebo group, CRP increased after steroid withdrawal at day 15 postrandomization. This was not evident in the AZD7624 group (Figure S5).

\section{Safety}

The most commonly reported AE was COPD, followed by dyspnea and cough. AZD7624 was well tolerated, although for the majority of reported categories of AEs (Table 4), the incidence was higher for participants in the AZD7624 treatment arm compared with placebo. Overall, 13 participants discontinued the investigational product owing to an AE: nine in the AZD7624 arm and four on placebo. The number of participants with SAEs was similar between the two treatment groups. One participant treated with AZD7624 died from cardiac arrest during the study. The event was not considered related to the study drug by the site physician.

\section{Discussion}

In this set of studies, we identified the $\mathrm{p} 38 \alpha$ isoform as being activated in patients with severe COPD in both lung macrophages and epithelial cells. We also identified p $38 \alpha$ as the predominant isoform activated in the human alveolar macrophage response to LPS stimulation in vitro and AZD7624 as a specific inhibitor of $\mathrm{p} 38 \alpha$ with less activity against p38 $\beta$. Furthermore, AZD7624 decreased LPS-stimulated cytokine release in human alveolar macrophages in vitro, and decreased systemic and lung inflammatory markers after inhaled LPS challenge. However, AZD7624 failed to provide any benefit in a 3 month COPD exacerbation study.

Several oral p38 inhibitors, including losmapimod (GlaxoSmithKline) and PH-797804 (Pfizer), both potent inhibitors of $\mathrm{p} 38$ MAPK $\alpha / \beta,{ }^{28,29}$ have been tested in COPD on top of a mix of background COPD therapies. These studies 


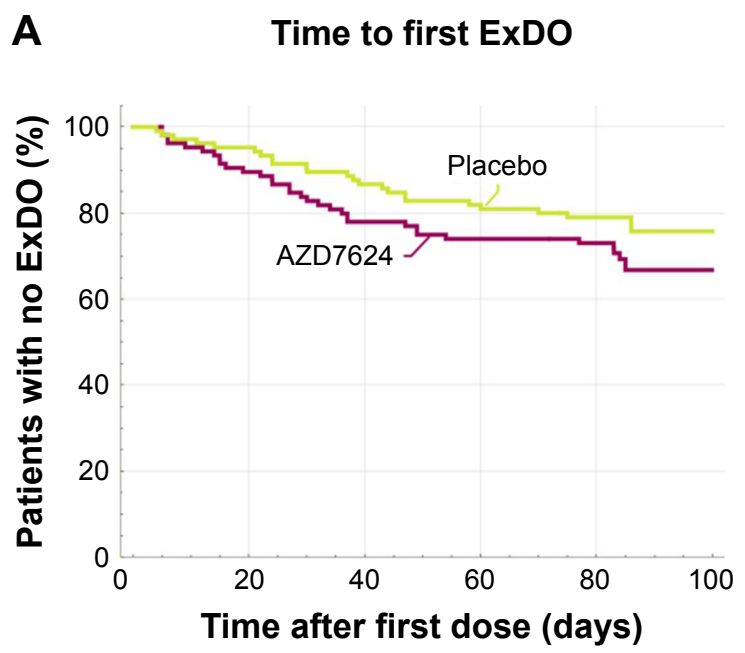

$\begin{array}{rllllll}\text { No at risk } & & & & & & \\ \text { AZD7624 } & 107 & 93 & 80 & 75 & 74 & 0 \\ \text { Placebo } & 105 & 100 & 91 & 84 & 79 & 3\end{array}$

C Time to first ExDO ( $\leq 2 \%$ eosinophils)

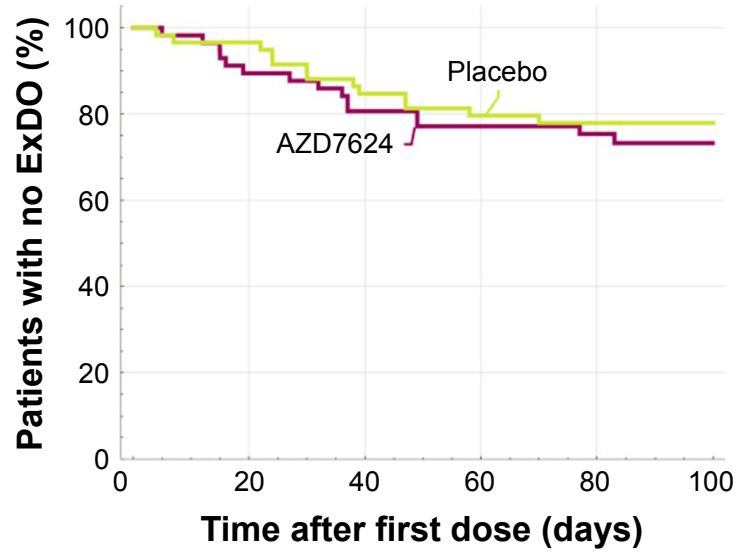

No at risk

$\begin{array}{rrrrrrr}\text { AZD7624 } & 58 & 51 & 46 & 44 & 43 & 0 \\ \text { Placebo } & 59 & 57 & 50 & 47 & 44 & 1\end{array}$

B

Time to first moderate or severe exacerbation

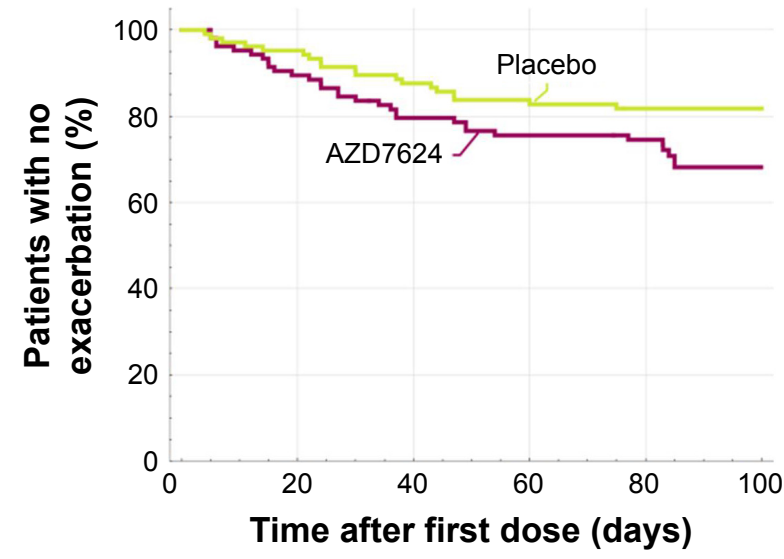

No at risk

$\begin{array}{rllllll}\text { AZD7624 } & 107 & 91 & 80 & 75 & 74 & 0 \\ \text { Placebo } & 105 & 100 & 91 & 83 & 79 & 2\end{array}$

D

Time to first ExDO (>2\% eosinophils)

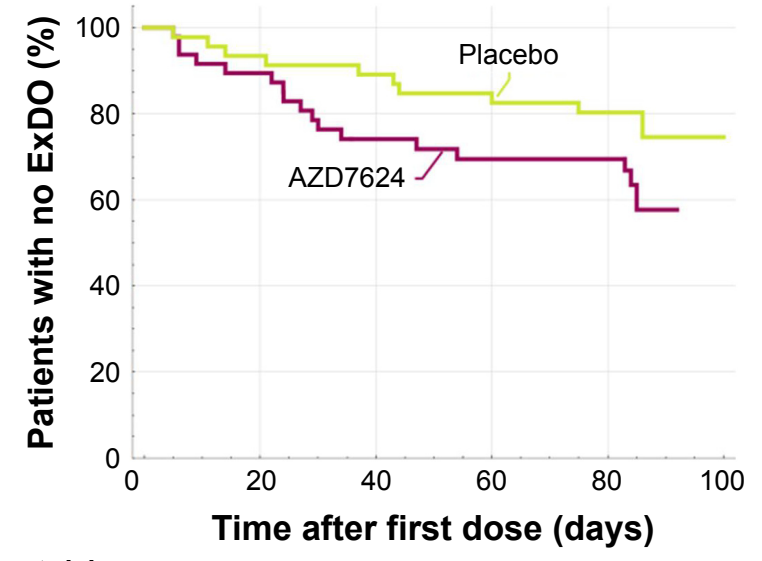

$\begin{array}{rllllll}\text { No at risk } & & & & & \\ \text { AZD7624 } & 48 & 41 & 33 & 30 & 30 & 0 \\ \text { Placebo } & 46 & 43 & 41 & 37 & 35 & 2\end{array}$

Figure 3 (A) Time to first event of ExDo ( $\mathrm{HR}=1.39,95 \% \mathrm{Cl} 0.8 \mathrm{I}, 2.40 ; p=0.24)$. (B) Time to first moderate or severe COPD exacerbation (HR =I.53, 95\% Cl 0.85, 2.76; $p=0.15)$. (C) Time to first event of ExDo $\leq 2 \%$ eosinophils ( $H R=0.93,95 \% \mathrm{Cl} 0.42,2.05 ; p=0.85)$. (D) Time to first event of ExDo $>2 \%$ eosinophils $(\mathrm{HR}=2.20,95 \% \mathrm{Cl} 0.95$, 5.09; $p=0.07$ ).

Abbreviation: ExDo, time to first event of moderate or severe COPD exacerbations or early dropout related to worsening of COPD symptoms.

have generally assessed lung function, not exacerbations, with no meaningful clinical improvement. ${ }^{16,17}$ Notably, a post hoc subgroup analysis of losmapimod suggested that p38 inhibition may reduce the frequency of exacerbations among participants with $\leq 2 \%$ blood eosinophils at baseline. ${ }^{30}$ The current study is the first published study of an inhaled p38 inhibitor in COPD patients with a primary exacerbation-based endpoint. The results of our study suggest that, although inhaled delivery of a p38 inhibitor can avoid the described systemic toxicities of oral p38 inhibitors, ${ }^{18}$ p38 inhibition provides no benefit with respect to reduction of exacerbations.
Although LPS challenge has been proposed as a model of airway inflammation that can be used to evaluate drugs for $\mathrm{COPD},{ }^{31-33}$ our results demonstrate the limited value of LPS challenge in predicting the benefit for COPD. In the current study, the findings that systemic biomarkers (CRP, IL-6, MIP-1 $\beta$ ) were decreased by AZD7624 after LPS challenge were not reproduced in COPD patients. The differential effect on CRP after steroid withdrawal suggested biological activity of AZD7624. However, this did not translate into benefit, as evidenced by an early higher exacerbation rate in the AZD7624 group, at a time when CRP was lower compared with the placebo (Figure S5). 
Table 3 Key secondary endpoints, COPD Proof of Principle study

\begin{tabular}{|c|c|c|c|}
\hline \multirow[t]{2}{*}{ Secondary endpoint } & \multirow{2}{*}{$\frac{\text { AZD7624 I.0 mg }\left(\mathrm{n}=107^{\mathrm{a}}\right)}{\text { Change from baseline }}$} & \multirow{2}{*}{$\frac{\text { Placebo }(n=105)}{\text { Change from baseline }}$} & \multirow{2}{*}{$\frac{\text { AZD7624 vs placebo }}{\text { p-value }^{\text {b }}}$} \\
\hline & & & \\
\hline Number of ExDo events & 40 & 29 & 0.25 \\
\hline Number of moderate or severe exacerbations & 38 & 24 & 0.13 \\
\hline $\begin{array}{l}\text { Number of moderate or severe exacerbations according } \\
\text { to Anthonisen criteria fulfilled }\end{array}$ & 17 & 15 & 0.75 \\
\hline $\begin{array}{l}\text { Number of symptom-defined exacerbations as defined } \\
\text { by EXACT daily dairy }\end{array}$ & 42 & 50 & 0.44 \\
\hline Post-bronchodilator FEV, (L) & $-0.003(0.26)$ & $-0.03(0.23)$ & 0.51 \\
\hline Post-bronchodilator FVC (L) & $-0.045(0.40)$ & $-0.018(0.39)$ & 0.05 \\
\hline $\mathrm{FEV}_{1} / \mathrm{FVC}$ & $0.04(0.06)$ & $-0.007(0.05)$ & 0.15 \\
\hline SGRQ-C & $-4.0(19.6)$ & $-5.1(17.8)$ & 0.52 \\
\hline TDI & $1.6(3.2)$ & $1.7(3.2)$ & 0.49 \\
\hline EXACT E-RS & $-0.2(6.4)$ & $-0.2(6.3)$ & 0.55 \\
\hline Use of reliever medication (average puffs per day) & $-0.13(2.27)$ & $-0.29(2.12)$ & 0.22 \\
\hline Night-time awakenings ( $\%$ of days during a 2 -week period) & $-8.98(32.53)$ & $-11.65(33.90)$ & 0.66 \\
\hline \multirow[t]{2}{*}{ Secondary endpoint } & \multicolumn{3}{|c|}{ AZD7624 $\left(n=107^{a}\right)$ vs placebo $(n=105)$} \\
\hline & HR & $95 \% \mathrm{Cl}$ & p-value ${ }^{b}$ \\
\hline $\begin{array}{l}\text { Time to first moderate or severe exacerbations according } \\
\text { to Anthonisen criteria fulfilled }\end{array}$ & 0.93 & $0.43,2.01$ & 0.85 \\
\hline $\begin{array}{l}\text { Time to symptom-defined exacerbations as defined } \\
\text { by EXACT daily dairy }\end{array}$ & 0.86 & $0.54,1.38$ & 0.54 \\
\hline
\end{tabular}

Notes: ane patient was excluded from the analysis following a review of protocol deviations owing to a lack of source data and Good Clinical Practice compliance. bMixed-effects repeated measures analysis, fitting treatment, country, LAMA maintenance treatment, visit, gender, smoking history, and treatment by visit interaction as fixed effects; patient as a random effect; and baseline assessment, age, BMI, and height as continuous covariates.

Abbreviations: BMI, body mass index; E-RS, Evaluating Respiratory Symptoms; ExDo, time to first event of moderate or severe COPD exacerbations or early dropout related to worsening of COPD symptoms; EXACT, EXAcerbations of Chronic pulmonary disease Tool; LAMA, long-acting muscarinic antagonist; SGRQ-C, St George's Respiratory Questionnaire for COPD Patients; TDI, Transition Dyspnea Index.

Prior studies of anti-inflammatories have shown superior effect in subgroups of COPD compared with limited benefit in unselected patient populations. Roflumilast did not show benefit in an unselected COPD patient population, ${ }^{34}$ but did benefit patients with chronic bronchitis ${ }^{12}$ and a CXCR2 ${ }^{35}$ inhibitor only showed benefit in active smokers. Evidence suggests that inhaled corticosteroids are more effective in a subset of COPD patients with higher baseline blood eosinophils. ${ }^{36}$ In the current study, not only did p38 inhibition fail to show benefit, but there was also a trend toward increased exacerbations in patients treated with AZD7624. This trend was largely observed in patients with baseline blood eosinophils $>2 \%$. Whereas other anti-inflammatory agents have only demonstrated benefit in subpopulations of COPD, our data suggest that some anti-inflammatories may have undesirable effects in COPD subpopulations. This stresses the importance of understanding the underlying biology and identifying subtypes of COPD so that effective therapy can be developed. Although significant attempts to categorize COPD patients according to radiographic findings and/or clinical findings are ongoing, ${ }^{35,37}$ the link to biology and pathogenesis remains poorly understood and more research is required.
Our study is limited, in that the design of the clinical COPD study contains a number of nonstandard elements designed to allow testing of exacerbations in a relatively small and short study. Although more research is required to validate this new study design, the absence of any signal in secondary endpoints supports the conclusions on the lack of efficacy of AZD7624. The lack of sputum biomarkers in the COPD study limited our ability to understand the specific biological effects of AZD7624 in the lung. The possibility of COPD subgroups which respond to $\mathrm{p} 38 \alpha$ inhibition cannot be ruled out, although the absence of any favorable subgroup response in the current study makes this unlikely.

In conclusion, although $\mathrm{p} 38 \alpha$ is upregulated in lungs of COPD patients, AZD7624, an isoform-specific inhaled p38 MAP kinase inhibitor, failed to show any benefit in patients with COPD. As COPD is one of the leading causes of mortality worldwide, new medicines are needed to help treat this expanding global health problem. Incomplete knowledge of the drivers of COPD and exacerbations continues to hamper drug development, and more research is needed to understand COPD and its subtypes of patients. 
Table 4 AEs and serious AEs, COPD Proof of Principle study ${ }^{a}$

\begin{tabular}{|c|c|c|c|}
\hline \multirow[t]{2}{*}{ Variable $^{b}$} & $\begin{array}{l}\text { AZD7624 } \\
1.0 \mathrm{mg} \\
\end{array}$ & Placebo & Total \\
\hline & $(n=108)$ & $(n=105)$ & $(\mathbf{N}=\mathbf{2} \mid \mathbf{3})$ \\
\hline Participants with $\geq 1 \%$ AEs & $71(65.7)$ & $48(45.7)$ & $119(55.9)$ \\
\hline \multicolumn{4}{|c|}{$A E$ that occurred in $\geq 2 \%$ of either treatment group } \\
\hline COPD & $31(28.7)$ & $23(21.9)$ & $54(25.4)$ \\
\hline Cough & $8(7.4)$ & $2(1.9)$ & $10(4.7)$ \\
\hline Dyspnea & $8(7.4)$ & $4(3.8)$ & $12(5.6)$ \\
\hline Dysgeusia & $5(4.6)$ & $\mathrm{I}(\mathrm{I} .0)$ & $6(2.8)$ \\
\hline Influenza & $5(4.6)$ & $3(2.9)$ & $8(3.8)$ \\
\hline Back pain & $4(3.7)$ & $\mathrm{I}(\mathrm{I} .0)$ & $5(2.3)$ \\
\hline Bronchitis & $4(3.7)$ & $2(1.9)$ & $6(2.8)$ \\
\hline Nasopharyngitis & $4(3.7)$ & $4(3.8)$ & $8(3.8)$ \\
\hline Fatigue & $3(2.8)$ & 0 & $3(1.4)$ \\
\hline Hypertension & $3(2.8)$ & $2(1.9)$ & $5(2.3)$ \\
\hline Productive cough & $3(2.8)$ & 0 & $3(1.4)$ \\
\hline Asthenia & $2(1.9)$ & $4(3.8)$ & $6(2.8)$ \\
\hline Sinusitis & I (0.9) & $3(2.9)$ & $4(1.9)$ \\
\hline Peripheral edema & 0 & $3(2.9)$ & $3(1.4)$ \\
\hline Pain in extremity & 0 & $3(2.9)$ & $3(1.4)$ \\
\hline Serious $A E$ & II (I0.2) & $11(10.5)$ & $22(10.3)$ \\
\hline Death & I (0.9) & 0 & $\mathrm{I}(0.5)$ \\
\hline $\begin{array}{l}\text { Participants who discontinued } \\
\text { because of an } A E\end{array}$ & $9(8.3)$ & $4(3.8)$ & $13(6.1)$ \\
\hline
\end{tabular}

Notes: Data are shown as number (\%) of participants. ${ }^{a}$ The safety analysis included patients who received a drug during the treatment period. 'Events were coded according to preferred terms in MedDRA, a standardized dictionary for clinical trials. $^{38}$

Abbreviation: $\mathrm{AE}$, adverse event.

\section{Author contributions}

PJ, MF, EE, and DMC were involved in the design, data analysis, and interpretation of the human Proof of Mechanism study and the Proof of Principle trial. KP and NK were involved in the design, data generation, and delineation of the relevant p38 isoforms. LS, GWC, and SG were involved in the design, data analysis, and interpretation of the human Proof of Mechanism study. NRP, MNB, SIR, and ZT were involved in the data analysis and interpretation of the human Proof of Mechanism study and the Proof of Principle trial. BJM, RAW, KK, CK, and RP were involved in the data analysis and interpretation of the Proof of Principle trial. DMC, SL, THM, and SA were involved in data interpretation for all of the studies reported in this manuscript. The first draft of the manuscript was written jointly by PJ, NRP, and DMC, who all equally contributed to the development of the manuscript. All authors contributed toward revising the paper and made the decision to submit the manuscript for publication. All authors agree to be accountable for all aspects of the work.

\section{Disclosure}

NRP, DMC, MF, ZT, SA, THM, SL, KP, NK, EE, CK, KK, RP, MNB, SIR, and PJ are AstraZeneca employees and hold stocks or shares in AstraZeneca. SL, SG, LS, and GWC no longer work for AstraZeneca or Quintiles Drug Research Unit at Guy's Hospital, but were employees of the companies at the time of this work. BJM is an advisory board member for AstraZeneca. No author received reimbursement for the preparation of this report. The authors report no other conflicts of interest in this work.

\section{References}

1. Hurst JR, Vestbo J, Anzueto A, et al. Susceptibility to exacerbation in chronic obstructive pulmonary disease. N Engl J Med. 2010;363(12): $1128-1138$

2. Anzueto A, Sethi S, Martinez FJ. Exacerbations of chronic obstructive pulmonary disease. Proc Am Thorac Soc. 2007;4(7):554-564.

3. Perera WR, Hurst JR, Wilkinson TMA, et al. Inflammatory changes, recovery and recurrence at COPD exacerbation. Eur Respir J. 2007; 29(3):527-534.

4. Niewoehner DE. The impact of severe exacerbations on quality of life and the clinical course of chronic obstructive pulmonary disease. Am J Med. 2006;119(10 Suppl 1):38-45.

5. Vestbo J, Hurd SS, Agustí AG, et al. Global strategy for the diagnosis, management, and prevention of chronic obstructive pulmonary disease. Am J Respir Crit Care Med. 2013;187(4):347-365.

6. Sethi S, Murphy TF. Infection in the pathogenesis and course of chronic obstructive pulmonary disease. N Engl J Med. 2008;359(22): 2355-2365.

7. Papi A, Bellettato CM, Braccioni F, et al. Infections and airway inflammation in chronic obstructive pulmonary disease severe exacerbations. Am J Respir Crit Care Med. 2006;173(10):1114-1121.

8. Groenewegen KH, Postma DS, Hop WCJ, Wielders PLML, Schlösser NJJ, Wouters EFM. Increased systemic inflammation is a risk factor for COPD exacerbations. Chest. 2008;133(2):350-357.

9. Thomsen M, Ingebrigtsen TS, Marott JL, et al. Inflammatory biomarkers and exacerbations in chronic obstructive pulmonary disease. JAMA. 2013;309(22):2353-2361.

10. Celli BR, Barnes PJ. Exacerbations of chronic obstructive pulmonary disease. Eur Respir J. 2007;29(6):1224-1238.

11. Ernst P, Saad N, Suissa S. Inhaled corticosteroids in COPD: the clinical evidence. Eur Respir J. 2015;45(2):525-537.

12. Martinez FJ, Calverley PMA, Goehring U-M, Brose M, Fabbri LM, Rabe KF. Effect of roflumilast on exacerbations in patients with severe chronic obstructive pulmonary disease uncontrolled by combination therapy (REACT): a multicentre randomised controlled trial. Lancet. 2015;385(9971):857-866.

13. Saklatvala J. The p38 MAP kinase pathway as a therapeutic target in inflammatory disease. Curr Opin Pharmacol. 2004;4(4):372-377.

14. Chung KF. p38 Mitogen-activated protein kinase pathways in asthma and COPD. Chest. 2011;139(6):1470-1479.

15. Renda T, Baraldo S, Pelaia G, et al. Increased activation of p 38 MAPK in COPD. Eur Respir J. 2008;31(1):62-69.

16. MacNee W, Allan RJ, Jones I, De Salvo MC, Tan LF. Efficacy and safety of the oral p38 inhibitor PH-797804 in chronic obstructive pulmonary disease: a randomised clinical trial. Thorax. 2013;68(8): 738-745.

17. Watz H, Barnacle H, Hartley BF, Chan R. Efficacy and safety of the p38 MAPK inhibitor losmapimod for patients with chronic obstructive pulmonary disease: a randomised, double-blind, placebo-controlled trial. Lancet Respir Med. 2014;2(1):63-72.

18. Fisk M, Gajendragadkar PR, Mäki-Petäjä KM, Wilkinson IB, Cheriyan J. Therapeutic potential of p38 MAP kinase inhibition in the management of cardiovascular disease. Am J Cardiovasc Drugs. 2014; 14(3):155-165.

19. From the Global Strategy for the Diagnosis, Management and Prevention of COPD, Global Initiative for Chronic Obstructive Lung Disease (GOLD) 2017. Available from: http://goldcopd.org 
20. Pizzichini E, Pizzichini MM, Efthimiadis A, et al. Indices of airway inflammation in induced sputum: reproducibility and validity of cell and fluid-phase measurements. Am J Respir Crit Care Med. 1996; 154(2):308-317.

21. Quanjer PH, Stanojevic S, Cole TJ, et al. Multi-ethnic reference values for spirometry for the 3-95-yr age range: the global lung function 2012 equations. Eur Respir J. 2012;40(6):1324-1343.

22. Jenkins CR, Celli B, Anderson JA, et al. Seasonality and determinants of moderate and severe COPD exacerbations in the TORCH study. Eur Respir J. 2012;39(1):38-45.

23. Ahrens RC, Teresi ME, Han SH, Donnell D, Vanden Burgt JA, Lux CR. Asthma stability after oral prednisone: a clinical model for comparing inhaled steroid potency. Am J Respir Crit Care Med. 2001;164(7): 1138-1145.

24. Calverley PM, Boonsawat W, Cseke Z, Zhong N, Peterson S, Olsson H. Maintenance therapy with budesonide and formoterol in chronic obstructive pulmonary disease. Eur Respir J. 2003;22(6):912-919.

25. Burge PS, Calverley PM, Jones PW, Spencer S, Anderson JA, Maslen TK. Randomised, double blind, placebo controlled study of fluticasone propionate in patients with moderate to severe chronic obstructive pulmonary disease: the ISOLDE trial. BMJ. 2000;320(7245):1297-1303.

26. Dransfield MT, Bourbeau J, Jones PW, et al. Once-daily inhaled fluticasone furoate and vilanterol versus vilanterol only for prevention of exacerbations of COPD: two replicate double-blind, parallel-group, randomised controlled trials. Lancet Respir Med. 2013;1(3):210-223.

27. Eriksson G, Calverley PM, Jenkins CR, et al. The effect of COPD severity and study duration on exacerbation outcome in randomized controlled trials. Int J Chron Obstruct Pulmon Dis. 2017;12:1457-1468.

28. Hope HR, Anderson GD, Burnette BL, et al. Anti-inflammatory properties of a novel N-phenyl pyridinone inhibitor of p38 mitogen-activated protein kinase: preclinical-to-clinical translation. J Pharmacol Exp Ther. 2009;331(3):882-895.

29. Aston NM, Bamborough P, Buckton JB, et al. p38 $\alpha$ mitogen-activated protein kinase inhibitors: optimization of a series of biphenylamides to give a molecule suitable for clinical progression. J Med Chem. 2009; 52(20):6257-6269.
30. Marks-Konczalik J, Costa M, Robertson J, McKie E, Yang S, Pascoe S A post-hoc subgroup analysis of data from a six month clinical trial comparing the efficacy and safety of losmapimod in moderate-severe COPD patients with $\leq 2 \%$ and $>2 \%$ blood eosinophils. Respir Med. 2015;109(7):860-869.

31. Aul R, Armstrong J, Duvoix A, et al. Inhaled LPS challenges in smokers: a study of pulmonary and systemic effects. Br J Clin Pharmacol. 2012;74(6):1023-1032.

32. Korsgren M, Linden M, Entwistle N, et al. Inhalation of LPS induces inflammatory airway responses mimicking characteristics of chronic obstructive pulmonary disease. Clin Physiol Funct Imaging. 2012;32(1):71-79.

33. Michel O, Dentener M, Cataldo D, et al. Evaluation of oral corticosteroids and phosphodiesterase-4 inhibitor on the acute inflammation induced by inhaled lipopolysaccharide in human. Pulm Pharmacol Ther. 2007;20(6):676-683.

34. Rennard SI, Calverley PM, Goehring UM, Bredenbroker D, Martinez FJ. Reduction of exacerbations by the PDE4 inhibitor roflumilast - the importance of defining different subsets of patients with COPD. Respir Res. 2011;12:18.

35. Rennard SI, Dale DC, Donohue JF, et al. CXCR2 Antagonist MK-7123. A phase 2 proof-of-concept trial for chronic obstructive pulmonary disease. Am J Respir Crit Care Med. 2015;191(9):1001-1011.

36. Pavord ID, Lettis S, Locantore N, et al. Blood eosinophils and inhaled corticosteroid/long-acting $\beta$-2 agonist efficacy in COPD. Thorax. 2016; 71(2):118-125.

37. Regan EA, Hokanson JE, Murphy JR, et al. Genetic Epidemiology of COPD (COPDGene) study design. COPD. 2010;7(1):32-43.

38. MedDRA [webpage on the Internet]. Medical Dictionary for Regulartory Activities; 2014: version 17.0. Available from: https:/www. meddra.org/how-to-use/support-documentation/english. Accessed February 1, 2018.
International Journal of COPD

\section{Publish your work in this journal}

The International Journal of COPD is an international, peer-reviewed journal of therapeutics and pharmacology focusing on concise rapid reporting of clinical studies and reviews in COPD. Special focus is given to the pathophysiological processes underlying the disease, intervention programs, patient focused education, and self management protocols.

\section{Dovepress}

This journal is indexed on PubMed Central, MedLine and CAS. The manuscript management system is completely online and includes a very quick and fair peer-review system, which is all easy to use. Visit http://www.dovepress.com/testimonials.php to read real quotes from published authors. 